\title{
ESTUDO ANATÔMICO DAS VIAS BILÍFERAS EM BÚFAI.OS (Bubalus Bubalis, L. 1758). SISTEMATIZAÇÃO DO Ramus principalis sinister
}

\author{
ANATOMICAL STUDY OF THE BILIARY DUCTS IN BUFFALOES (Bubalus bubalis, L. 1758). INTRA-IIEPATIC \\ DISTRIBUTION OF THE Ramus principalis sinister
}

Wilson Machado de SOUZA'; Nair Trevisan Machado SOLZA²; Maria Angélica MIGIJNO'; Roberto CARVALHAL ${ }^{4}$

\begin{abstract}
RESUMO
Estabeleceu-se a sistematização do ramus principalis sinister, um dos componentes das vias bilíferas intra-hepáticas em 40 búfilos têmeas da raça Jaffarabadi adultas. Através do ductus choledocus, as vias bilíferas de cada animal foram injetadas com látex Neoprene 650 e Celobar. radiografadas e dissecadas após fixaçāo $\mathrm{em}$ solução aquosa de formol a $10 \%$ O ramus principalis sinister é formado de maneira geral pelos seus componentes: ramus medialis lobi sinistri. truncus intermediamedialis. truncus intermediolateralis. ramus lateralis lobi sinistri e ramus lobi quadrati. além de afluentes inominados oriundos do lobus caudatus (pars supraportalis), Iohus sinister (territórios: medial, intermédio e lateral), lobus quadratus e lohus dexter. Foram observados em alguns órgãos determinados componentes do sistema do ramus principalis sinister relacionando-se entre si ou com a vesica fellea.
\end{abstract}

UNITERMOS: Anatomia Animal; Búfalos; Fígado; Ductos biliares

\section{INTRODUÇÃO E LITERATURA}

Neste trabalho apresentamos resultados atinentes às vias bilíferas intra-hepáticas, particularizando-se as componentes do ramus principalis sinister, de búfalos, comparando as suas disposições, com os resultados obtidos nos bovinos.

Entre os tratados de anatomia animal, encontramos algumas informações, embora genéricas, sobre o assunto, relatadas por GETTY2 (1975), segundo as quais, pequenos ductos bilíferos confluem de diferentes maneiras, para formar os ductos hepáticos dircito e esquerdo.

Em trabalhos especializados sobre as vias excretoras do fígado dos ruminantes, a saber, os de OTTAVIANI ${ }^{5}$ (1933); JABLAN-PANTIC ${ }^{3}$ (1963); BASTOS-NETO; PRADA (1983); SOUZA et al. ${ }^{6.7}$ (1989), que trabalharam com algumas espécies destes animais, há dados que se referem ao comportamento destas vias e que discutiremos adiante.

O objetivo do trabalho foi sistematizar a distribuição das vias bilíferas no parênquima do fígado de búfalos.

\section{MATERIAI, F MÉTODO}

Neste estudo utilizamos 40 peças, retiradas de fêmeas adultas de búfalos da raça Jaffarabadi, com idade aproximada entre 4 e 6 anos, procedentes de diversas regiões de criação dos Estados de São Paulo e Minas Gerais e abatidas nos frigoríficos: Taquaritinga em Taquaritinga; Oranges em Sertãozinho e Vale da Prata em São João da Boa Vista, Estado de São Paulo.

Cada uma destas preparações constava, depois de adequada redução do fígado e porção do duodeno correspondente à localização da papila duodenal maior. Realizamos, cntão, a abertura da porção do trato intestinal, ao longo de sua margem livre para, a seguir esvaziarmos, o máximo possível, as vias extra-hepáticas, de modo particular, a vesica fellea, mediante suaves massagens.

Efetuamos em seguida nestes 40 órgãos a canalização do ductus choledocus injetando, deste modo, as vias bilíferas com látex Neoprene 650, adicionado ao "Celobar", na proporção de $50 \%$. Depois deste procedimento, os fígados foram radiografados, fixados em solução aquosa de formol a $10 \%$, dissecados pela face visceral e esquematizados, sendo

1-Professor Adjunto - Faculdade de Medicina Veterinária da UNESP - "campus" de Araçatubu

2-Técnico Especializado - Faculdade de Medicina Veterinária da L"NESP - "campus" de Araçatuba

3-Professor Associado - Faculdade de Medicina Veterinária e 7.ontecnia da USP

4-Professor Assistente - Faculdade de Medicina Veterinária da UNESP - "campus" de Araçatuba 
SOUZA, W. M.: SOUY.A. N. T. M.: MIGL_INO, M. A.; CARVALHAI., R. Fstudo anatomico das vias bilíferas cm bútalos (Bulbalus bubalis. I.. I758)

Sistematizạ̧ão do ramus principalis sinjster, Braz. J. vet. Res. anim. Sci.. São Paulo, v. 31, n. 1, p. 19-23. 1994.

ainda, algumas destas preparaçōes, fotografadas.

Na exposição dos resultados, admitimos a nomenclatura sugerida por JABLAN-PANTIC (1963).

Tendo em vista que o fígado dos búfalos não é lobado, adotamos a divisão sugerida por NICKEL et al. ${ }^{4}$ (1973), relativamente aos animais de um modo geral, em três partes (esquerda, intermédia e direita), com o auxílio de duas linhas imaginárias, traçadas sobre a face visceral do órgão. A primeira delas tem como pontos de referência a impressão esofágica a a incisura determinada pelo ligamento redondo, enquanto a segunda atinge o ponto de cruzamento da veia cava caudal com a margem arredondada do fígado, alcançando ventralmente a fossa determinada pela vesícula biliar. Este último reparo não levamos em conta, pois, nos búfalos, à semelhança dos bovinos, a vesica fellea é pendente, sendo a sua fossa bastante redurida. correspondendo somente à pequena parte do colo e, além deste fato. mostra o seu eixo maior sempre orientado numa mesma direção. Por outro lado, nestes animais, o ductus cysticus mostra-se, em toda a sua extensão, inserido no parênquima. determinando, deste modo, naturalmente, a divisão almejada. Desta maneira, a segunda linha por nós relerida sobrepóe-se em parte a este ducto, atingindo o cruzamento mencionado pelos autores, descrevendo quase sempre trajelo curvo, com a concavidade voltada para o lado direito, com o qual se relaciona. Lntre estas duas linhas identificamos a porção intermédia. constituida pelas porçōes infraportal, referente ao lobus quadratus e supraportal, componente do lobus caudatus.

\section{RESULTADOS}

O estudo efeluado nas vias bilíferas intra-hepáticas, em 40) fígados de fêmeass adultas de búfalos da raça Jallarabadi, com idade variando entre 3 e 6 anos, levou-nos a observar que estas vias apresentam a seguinte composição:

formam, de maneira geral, o sistema do ramus principalis sinister, além dos componentes: ramus medialis lobi sinistri. truncus intermediomedialis, truncus intermediolateralis. ramus lateralis lobi sinistri c ramus lobi quadrati, afluentes inominados, oriundos do lobus caudatus (pars supraportalis), lobus sinister (territórios medial, intermédio e lateral), lobus quadratus e lobus dexter.

O ramus principalis sinister mostra-se, uma ve\% formado, sempre al recolher tributários vindos, ao mesmo tempo do lobus quadratus e do lobus caudatus (pars supraportalis). 31 vezes $(77.5 \%)$ : apenas do lobus quadratus, 5 vezes $(12,5 \%)$; simultancamente dolobus sinister (território medial e lateral), lobus caudatus (pars supraportalis) e do lobus quadratus. 3 vezes $(7,5 \%)$ : concomitantemente do lobus sinister (território medial), lobus caudatus (pars supraportalis), lobus quadratus e do lobus dexter (território medial), 1 vez $(2,5 \%)$.

Os territórios medial, intermédio e lateral do lobus sinister. mostram como principais ductos escoadores, por ordem: o ramus medialis lobi sinistri, otruncus intermediomedialis o truncus intermediolateralis, o ramus lobi sinistri e o ramus lobi quadrati. vias às quais se somam diversos elementos inominados, procedentes dos referidos lobos e também do lobus caudatus (pars supraportalis), além dos rami intermedii lobi sinistri, provenientes do território intermédio do lobus sinister, não concorrentes para a formação dos releridos troncos.

Tratos de calibre e comportamento variảveis foram observados, em alguns órgãos ( $10 \%$ ), a relacionarem por anastomose. determinados componentes do sistema do ramus principalis sinister entre si, ou estes à vesica fellè.

\section{COMENTÁRIOS}

Os resultados agora vistos, com referência às vias bilíferas destes bubalinos, permitem um cotejo com aqueles já verificados em outros ruminantes. Deste modo, relativamente à composição do ramus principalis sinister, JABLANPANTIC (1963), assinala-o, nos bovinos, constituído pelo ramus processi papillaris, ramus lobi quadrati, ramus lateralis lobi sinistri, ramus medialis lobi sinistri c rami medii lobi sinistri; de outra parte, lanto BASTOS-NETO: PRAIA' (1983), nos bovinos azcbuados, como SOUZA el al. ${ }^{6.7}$ (1989) nos animais Nelore, observaram comportamento semelhante. mas fizeram algumas ressalvas nesta nomenclatura, com as quais os resultados agora assinalados nestes búfalos permitem-nos concordar, ou seja, os rami medii lobi sinistri, passam a ser designados como rami intermedii lobi sinistri, sendo vistos, a comporem constantemente, dois contigentes, o truncus intermediomedialis a o truncus intermediolateralis, além de tomarem parte da composição de outras vias. Outro aspecto a salientar é que JABLANPANTIC (1963) identificou, a fazer parte do sistema do ramus principalis sinister, o ramus processi papillaris. fato não caracterizado nos bovinos, quer por BASTOSNETO; PRADA' (1983) nos azebuados, quer por SOUZA et al. ${ }^{7}$ ( 1989 ) nos Nelore, pois, conforme estes autores. no fígado destes animais não se define um lípico processo papilar e fato semelhante pode agora ser assinalado nos búfalos.

Tanı) BASTOS-NETO; PRADA' (1983), nos bovinos azcbuados como SOUZA et al. ${ }^{7}$ (1989) nos animais Nelore, encontraram a fazer parte do sistema do ramus principalis sinister, o ramus medialis lobi sinistri, o truncus intermediomedialis, ot runcus intermediolateralis, oramus lateralis lobi sinistri e o ramus lobi quadrati. além de coletores inominados oriundos do lobus caudatus (pars 
Supraportalis). lobus sinister (territórios medial, intermédio e lateral) c lobus quadratus, os primeiros autores surpreendem a eventual participação $(6,7 \%)$ do ramus medialis lobi dextri, na composição desta via. Em relação aos búlalos, agora observamos, a comporem constantemente o aludido sistema, o ramus medialis lobi sinistri, o truncus intermediomedialis, o truncus intermediolateralis, e o ramus lateralis lobi sinistri. Além destes, lomam parte no siste $(87,5 \%)$, o ramus medialis lobi dextri, o ramus dorsalis lobi dextri c o ramus processi caudati $(7,5 \%)$. Com respeito às contribuições inominadas, relacionadas ao ramus principalis sinister, verificamos estas vias, nos búfalos, procedentes do lobus caudatus (pars supraportalis), lobus sinister (territórios imedial, internédio e lateral) e lobus quadratus; estes contigentes não são referidos por JABLANPANTIC $(1963) \mathrm{cm}^{3}$ seu trabalho, porém já loram assinalados em grande número, lanto por BASTOS-NETO; PRADA' (1983) como por SOUZA cl al. ${ }^{7}(1989)$, nos animais por cles pesquisados

A seu turno GETTY2 (1975) alude pequenos ductos bilíferos se reunindo de distintas manciras para compor os ductos hepáticos direito e esquerdo. Estes contingentes porém. nâo sabemos se correspondem aos que agora referimos. De outra parte. OTTAVIANI' (1933), não estabelece objelivamente a exata conceituaçāo dos afluentes por ele designados de primários, secundários e terciários. mas indica o ducto bilílero do lobo esquerdo, ora denominado à semelhança de J A BLANPANTIC $^{3}$ (1963), de ramus principalis sinister. a ressaltar de variável número destes tributários. Provavelmente os afluentes terciários releridos por OTTAVIANI ${ }^{5}$ (1933), correspondem aos elementos inominados oriundos do lobus sinister, lobus caudatus c lobus quadratus, vistos a comporem oramus principalis sinister tanto nos bovinos azcbuados e Nelore como nos búfalos.

Considerando ocomportamento doramus principalis sinister em relação aos seus eventuais tributários. BASTOS-NETO; PRADA' (1983), observam-no livre de aferentes em algumas peças $(10 \%)$, fato semelhante ao consignalo por OTTAVIANI ${ }^{5}$ (1933), quando tais coletores surgem muito curtos, mas não registrado por SOU\%A et al. ? (1989) nos bovinos Nelore, onde o referido ducto é visto sempre a receber tributários; resultado semelhante verificamos agora nos búfalos. Certamente, os tributários oriundos simultancamente do lobus caudatus (pars supraportalis) e do lobus quadratus, assinalados por BASTOS-NET(); PRADA' (1983) em 30\% dos fígados, e por SOUZA el al. ${ }^{7}$ (1989) em 73,3\% das preparações e agora consignados na maioria das glândulas estudadas nos búfalos $(77,5 \%)$ equivalem aos alluentes primários dorsais e ventrais previamente associados, relerides por ()TTA VIANI (1933), bem como os componentes oriundos unicamente do lobus caudatus (pars supraportalis) vistos a alluírem ao ramus principalis sinister, nos bovinos azebuados $(20 \%)$, nos Nelore $(13,3 \%)$ e ora patenteado nos fígados dos búfalos em rara oportunidade $(2,5 \%)$. Talvez correspondam aos afluentes primários dorsais referidos pelo autor.

Finalmente, são vistos nos fígados dos búfalos (10\%), a relacionarem-se por conlluência, alguns dos componentes do ramus principalis sinister cntre si, ou estes à vesica fellea. Tal ocorrência foi assinalada em um maior número de glândulas $(16,7 \%)$ nos animais azebuados (BASTOS-NETO; PRADA $\left.^{\prime} 1983\right)$ e na maior parte delas $(73,3 \%)$ nos animais Nelore, onde os relacionados com a vesica fellea se comportam como tirantes, participando no mecanismo de suslentação da mesma (SOUZA cl al. ${ }^{6.7}, 1989$ ).

\section{CONCLUSÕES}

1. No búfalo da raça Jaffarabadi o ramus principalis sinister é formado pelo ramus medialis lobi sinistri. truncus intermediomedialis, truncus intermediolateralis, ramus lateralis lobi sinistri, ramus lobi quadrati, e por afluentes inominados oriundos do lobus caudatus (pars supraportalis). lobus sinister (territórios medial, intermédio c lateral), lobus quadratus e lobus dexter.

2. () ramus principalis sinister recebe tributários vindos simultancamente dolobus quadratus e dolobus caudatus $(77,5 \%)$, apenas do lobus quadratus $(12,5 \%)$, simultaneamente dos lobus sinister. lobus caudatus c lobus quad ratus $(7,5 \%)$; c concomitantemente dolobus sinister, lobus caudatus, lobus quadratus c lobus dexter (2,5\%).

3. Tratos de calibre e comportamento variáveis loram observados em alguns órgãos $(10 \%)$, a relacionarem por confluência determinados componentes do sistema do ramus principalis sinister entre si, ou estes à vesica fellea. 
SOUZA, W. M.; SOUZA, N. T. M.; MIGI.INO. M. A.; CARVALHAI., R. Listudo inatômico das vias bilíferas em búfialos (Bubalus bubalis. I.. 1758). Sistematização do ramus principalis sinister. Braz. J. vet. Res. anim. Sci.. Sāo Paulo. v. 31. n. 1. p. 19-23. 1994.

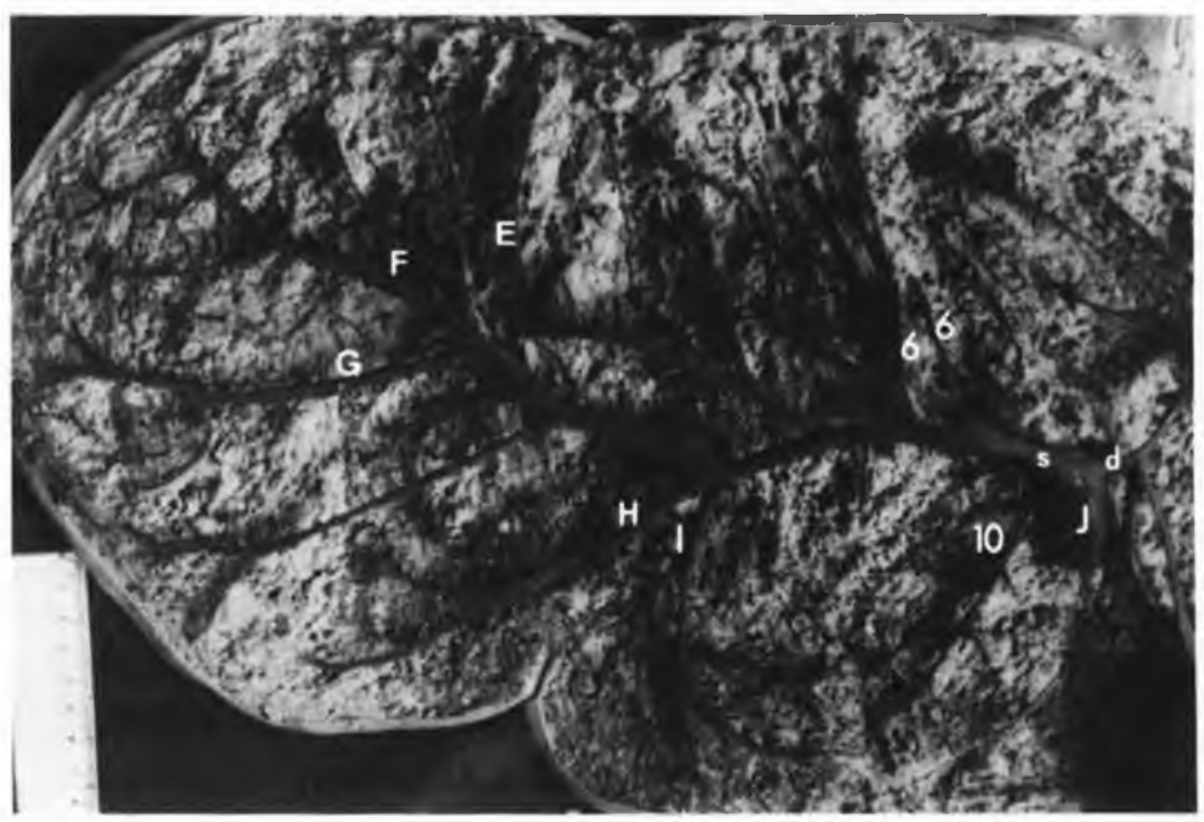

FICI:RA 1

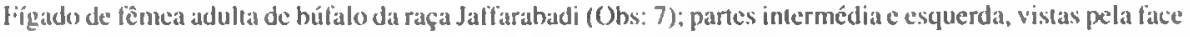
visceral. Reduçāo de aproximadamente., 2.5 veses. Oductus choledocus ( J) surgè da confluênciáa do ramus principalis dexter (d) e do ramus principalis sinister (s). O último destes forma-se pela convergêncjă sucessiva do truncus intermediomedialis (F) mais truncus intermediolaterais (G). ramus lateralis lobi sinistri (H), ramus lubi quadrati (I), ramus medialis lobi sinistri (E). O ramus principalis sinister (s). depojs de lormado, acolhe em seu percurso contingentes inominados oriundos da pars supraportalis do lolous caudatus (6) e do lobus quadratus ( (1)).

\section{SUMMARY}

The distribution of the ramus principalis sinister of the biliary ducts of 40 adult females of Jaffarabadi breed buffaloes wals studied after latex Neopreme 650 and Celohar injections through the ductus choledochus. Following fixation in $10 \%$ formaldehyde solution, radiograms were taken from the specimens and then dissected. The ramus principalis sinister is formed usually by several roots. ramus medialis Iobi sinistri, truncus intermediomedialis, truncus intermediolateralis. ramus lateralis lobi sinistri. In addition. innominate tribularies originating from the lobus caudatus (pars supraportalis) lobus sinister (medial, intermediate and latcral teritories), lobus (quadratus and lobus dexter. In a few cases the ramus principalis sinister had components inter-related or related to the vesica fellea

UNITERMS: Animal Anatomy; Buffaloes: Liver; Bile ducts

\section{REFERÊNCIAS BIBLIOGRÁFICAS}

)1-BASTOS NETO, I.P.; PRADA, I.L.S. Contribuição ao estudo das vias bilíleras de bovinos azebuados. Sistematização do ramus principalis sinister. Rev. lac. Med. Vet. Zootec. Univ. S. Paulo. v.20, p.23-7, 1983.

12-GETTY. R.Sisson and Grossman's. The anatomy of the domestic animals. 5.ed. Philadelphia, W.B. Saunders. 1975. v.1, p.913.
03-JABLAN-PANTIC, O. Characteristics and comparative ratios of intrahepatic bile duct in domestic animals. Acta vet., Beograd, v.13, n.3/4, p.3-14, 1963.

04-NICKEL, R.; SCHUMMER, A.; SEIFERLE, E.; SACK, W.O. The viscera of the domestic mammals. Berlin, Paul Parey. 1973. p.178. 
SOUZA, W. M.; SOUZA, N. T. M.; MIGLINO, M. A.; CARVALHAL, R. Estudo anatômico das vias bilíferas em búfalos (Bubalus bubalis, L. 1758). Sistematização do ramus principalis sinister. Braz. J. vet. Res. anim. Sci., Sảo Paulo, v. 31, n.1, p. 19-23, 1994.

05-OTTAVIANI, G. Ricerca radiografiche comparative sulle vie bilifere intraepatiche.Atti Ist. Veneto Sci., Venezia, v.92, p.1065-127, 1933.

06-SOUZA, W.M.; PRADA, I.L.S.; SOUZA, N.T.M. Estudo anatômico das vias bilíferas em bovinos da raça Nelore. I.Comportamento do ductus choledocus, ductus cysticus e vesica fellea. Tratos anastomóticos. Rev. Fac. Med. Vet. Zootec. Univ. S. Paulo, v.26, p.153-63, 1989.
07-SOUZA, W.M.; PRADA, I.L.S.; SOUZA, N.T.M. Estudo anatômico das vias bilíferas em bovinos da raça Nelore. II. Sistematização doramus principalis dexter e do ramus principalis sinister. Rev. Fac. Med. Vet. Zootec. Univ. S. Paulo, v. 26, p.165-76, 1989.

Recebido para publicação em 05/04/93 Aprovado para publicação em 19/10/93 